CAHIERS DE

NARRATOLOGIE

\section{Cahiers de Narratologie}

Analyse et théorie narratives

29 | 2015

Street Art 1

\title{
Ernest Pignon-Ernest : rencontre du troisième type
}

Transfiguration d'un lieu par la figure

Karin Wackers-Espinosa

\section{OpenEdition}

\section{Journals}

Electronic version

URL: http://journals.openedition.org/narratologie/7434

DOI: $10.4000 /$ narratologie. 7434

ISSN: 1765-307X

Publisher

LIRCES

Electronic reference

Karin Wackers-Espinosa, «Ernest Pignon-Ernest : rencontre du troisième type », Cahiers de Narratologie

[Online], 29 | 2015, Online since 28 January 2016, connection on 03 May 2019. URL : http://

journals.openedition.org/narratologie/7434; DOI : 10.4000/narratologie.7434

This text was automatically generated on 3 May 2019.

Article L.111-1 du Code de la propriété intellectuelle. 


\section{Ernest Pignon-Ernest : rencontre du troisième type}

Transfiguration d'un lieu par la figure

Karin Wackers-Espinosa

EDITOR'S NOTE

Lecture en annexe : Parcours romain : Pasolini, dans la succession d'une figure identique inlassablement déclinée.

1 Dès les années Soixante, quand l'appellation Street Art ne s'impose pas encore, Ernest Pignon-Ernest colle déjà, sans détours, des figures de poètes engagés, qui n'ont jamais hésité, à l'instar de Pier Paolo Pasolini, "à jeter leur corps dans la lutte ${ }^{1}$ ». Simultanément, il affirme aussi les contours anonymes de victimes des lois impitoyables du travail, de l'émigration; des corps écartelés, endurés d'une absolue souffrance.

2 Pour nourrir ces portraits, pour en trouver les traits justes, Ernest Pignon-Ernest convoque tour à tour Arthur Rimbaud, Vladimir Maïakovski, Antonin Artaud, Robert Desnos, Pablo Neruda, Jean Genet, Mahmoud Darwich, Pier Paolo Pasolini. Il laisse d'abord leurs mots errer dans sa tête, attend que surgisse face à lui un visage, un corps. De ces longs voyages, de ces errances volontaires, il revient dans son atelier et dessine. Il accumule les esquisses, décline à l'épuisement les formes jusqu'à n'en garder qu'une et la reproduire à l'envi. (Fig. 1) Il descend alors dans la rue. Là où l'espace fait écho aux mots, aux cris, il laisse la figure dire sans voix. 
Fig. 1

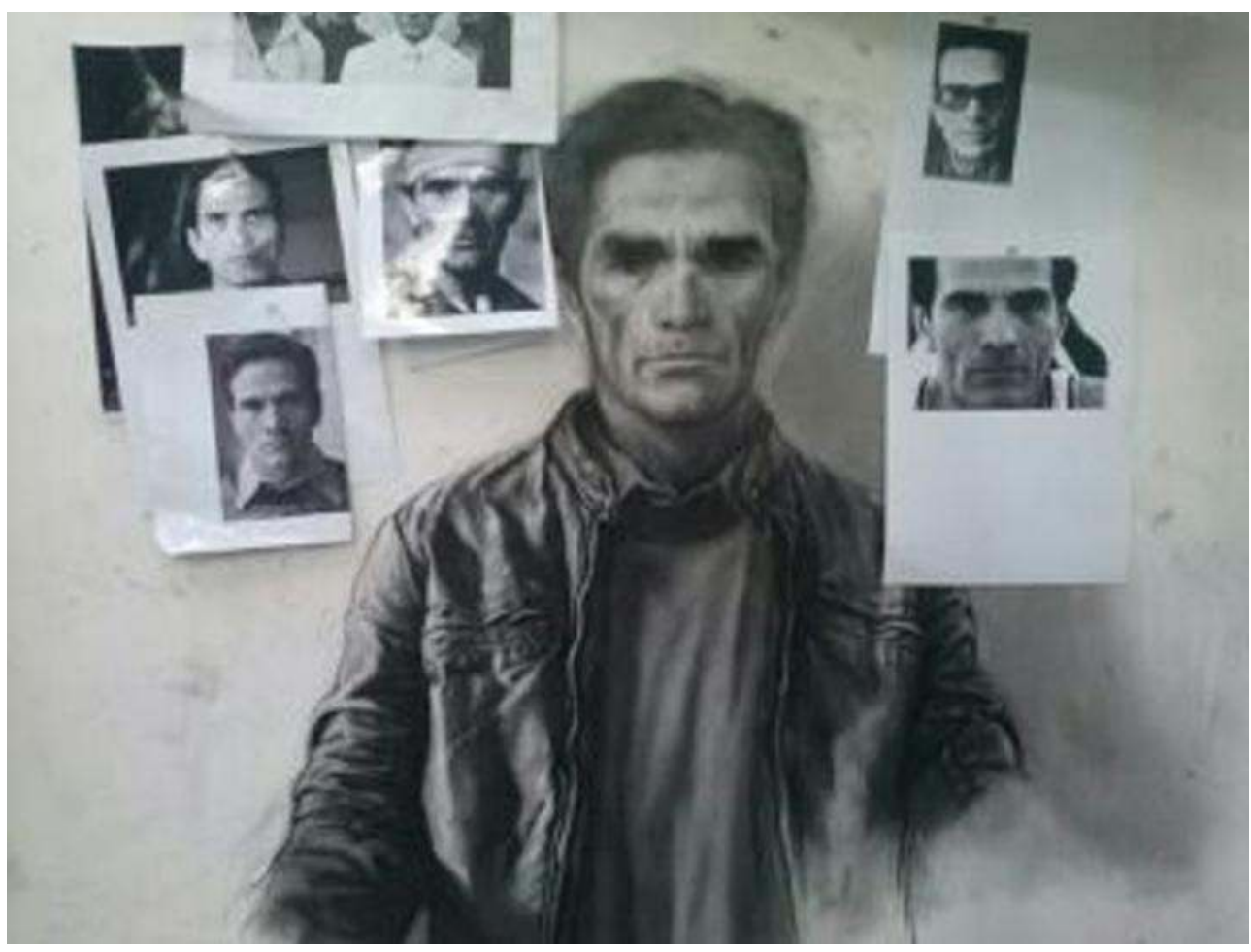

3 Depuis 1966, Ernest Pignon-Ernest oblige à penser autrement l'espace public, ouvert à tous, gratuitement. La rue est sa " palette ». Tout y est contenu pour l'amener au geste du dessin. Le choix d'un support-papier fragile (des rouleaux entiers de papier-journal vierge fournis par La Marseillaise) rend l'œuvre encore plus ultime. Ernest Pignon-Ernest ne pense pas la création pour l'éternité.

4 Son cheminement est multiple : il sourd de rencontres humaines, poétiques, citoyennes.

\section{Rencontre du premier type : Ernest Pignon-Ernest et le lieu}

5 Chaque lieu détient sa dramaturgie propre, soumise aux règles de la narration qui régissent son histoire profonde, aristotélicienne dans le fait même qu'elle repose en permanence sur des rapports de causalité, sur les conflits. Une ville, pas n'importe laquelle. Un lieu à habiter poétiquement, dans une vision romantique de cette seule façon d'être au monde par la poésie, douloureuse, impure, dans la vie comme dans la mort. L'artiste se nourrit, nourrit son œuvre des lieux mêmes, des murs, des parois, qui feraient écran à l'indicible.

Dans sa démarche première, la rencontre avec un lieu précède l'acte de création. Le lieu fait situation. Parce qu'il est plein, bien avant d'y installer ses figures de papier. Le lieu est un espace clos, bordé, mais qui reste ouvert à l'autre. Matériellement, il peut être circonscrit, fermé, défini. Ontologiquement, il est infini, par la mémoire qu'il porte et qui l'habite depuis sa création, et avant, en remontant jusqu'à sa source, d'avant son existence visible, tangible. Toujours redéfinissable, toujours à envisager autrement, ailleurs. (Fig. 2) 
Fig. 2

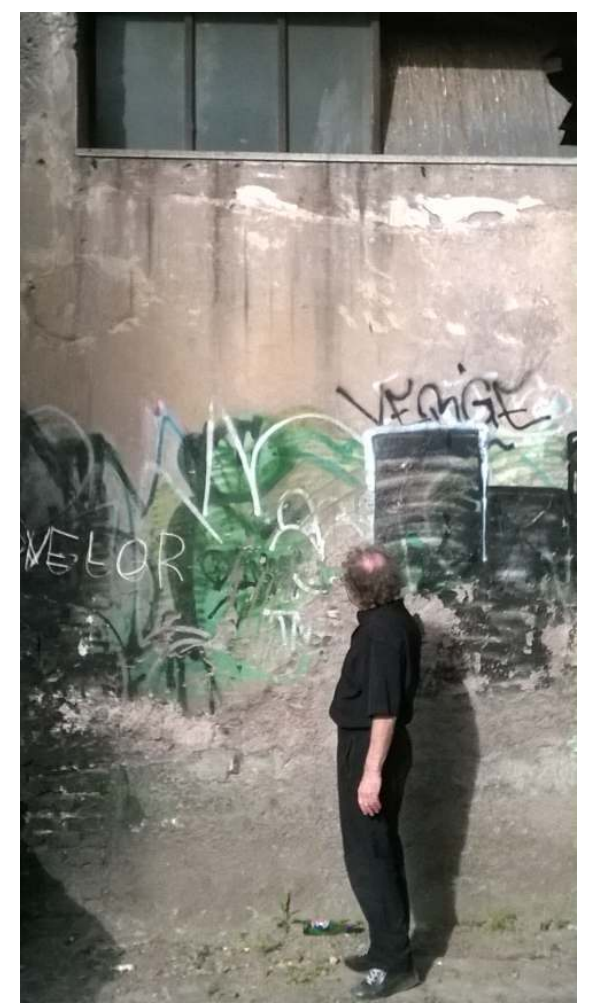

7 À cet égard, Bernard Salignon écrit que « l'architecture rencontre le corps, là où, séparés, ils partagent cet au-delà, cet infini qui déclôt leur limite vers l'illimité là où l'origine reste encore comme irrésolution en œuvre dans l'œuvre ${ }^{2} »$. Ce qui intéresse l'artiste, ce qui suscite le dessin et son installation à une place choisie, c'est ce lieu porteur d'autre chose, qui n'est pas immédiatement perceptible. Cette chose invisible n'est pas là, a priori. Il y a un lieu d'avant le lieu, celui qui est à l'origine du lieu présent, qui le précède, première strate d'une mémoire qui disparaîtra pour révéler l'invisible, devra s'effacer sans s'anéantir. Ce lieu n'est pas le néant. Il est un vide, un point de soubassement, le fondement de tous les lieux à venir.

$\mathrm{Au}$ cours de ses déambulations, de jour comme de nuit, Ernest Pignon-Ernest se laisse envahir, submerger par une perception sensible, entre vision et émotion. Il apprivoise humainement le lieu. Il passe sa main sur la paroi, sur les irrégularités, sur les graffitis, les tags. Il croise les gens, leur regard. Il les écoute parler, chanter et se laisse toucher par la musique de leur langue. (Fig. 3) Il repère les odeurs. Il pénètre dans l'existence même $\mathrm{du}$ lieu et de son logos, ce langage qui lui est propre, là où l'œuvre reste toujours à l'œuvre, où tous les lieux et les êtres restent à venir. Le lieu choisi par Ernest PignonErnest pour être à l'origine des dessins détient cette dimension universelle. Il appartient à tous les hommes et toutes les femmes qui en foulent le sol. Il a le passé pour soubassement, jusqu'aux tréfonds de la Mémoire de la terre, le présent pour combler un vide qui déjà n'est plus, un futur proche, moment précis où l'espace existe à nouveau pour d'autres que soi. (Fig. 4) 
Fig. 3

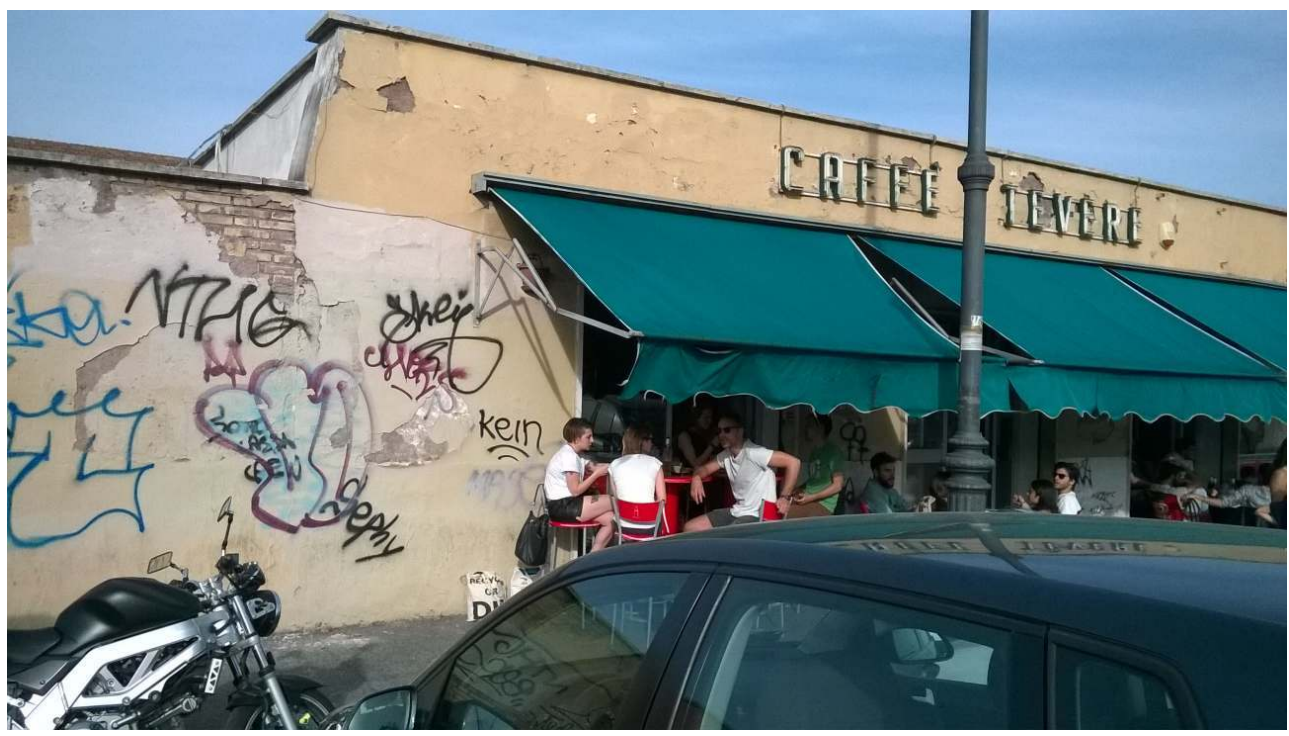

Fig. 4

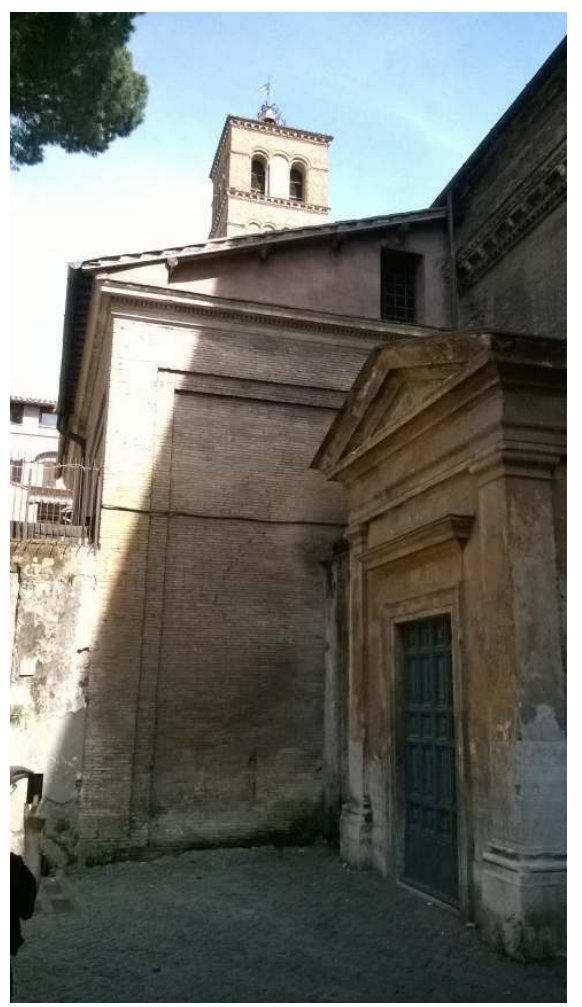

9 Et ce lieu rencontrera ensuite la figure, en épousera les contours, la renverra à son origine, éternel mystère révélé aux passants regardeurs.

10 La ville, en son tissu vivant et tactile, est comme un gigantesque dépôt d'images, d'images souvent perdues qui n'appartiennent qu'à la mémoire du passant et qui stagnent, en attente, comme une réserve proustienne que le pas longe et parfois éveille ${ }^{3}$. 


\section{Rencontre du deuxième type : la figure et le lieu}

11 Il n'y a pas de lieu anodin.

12 Le lieu naît de la main humaine, d'un effet/cause naturels. Il devient visible, tangible. (Fig. 5)

Fig. 5

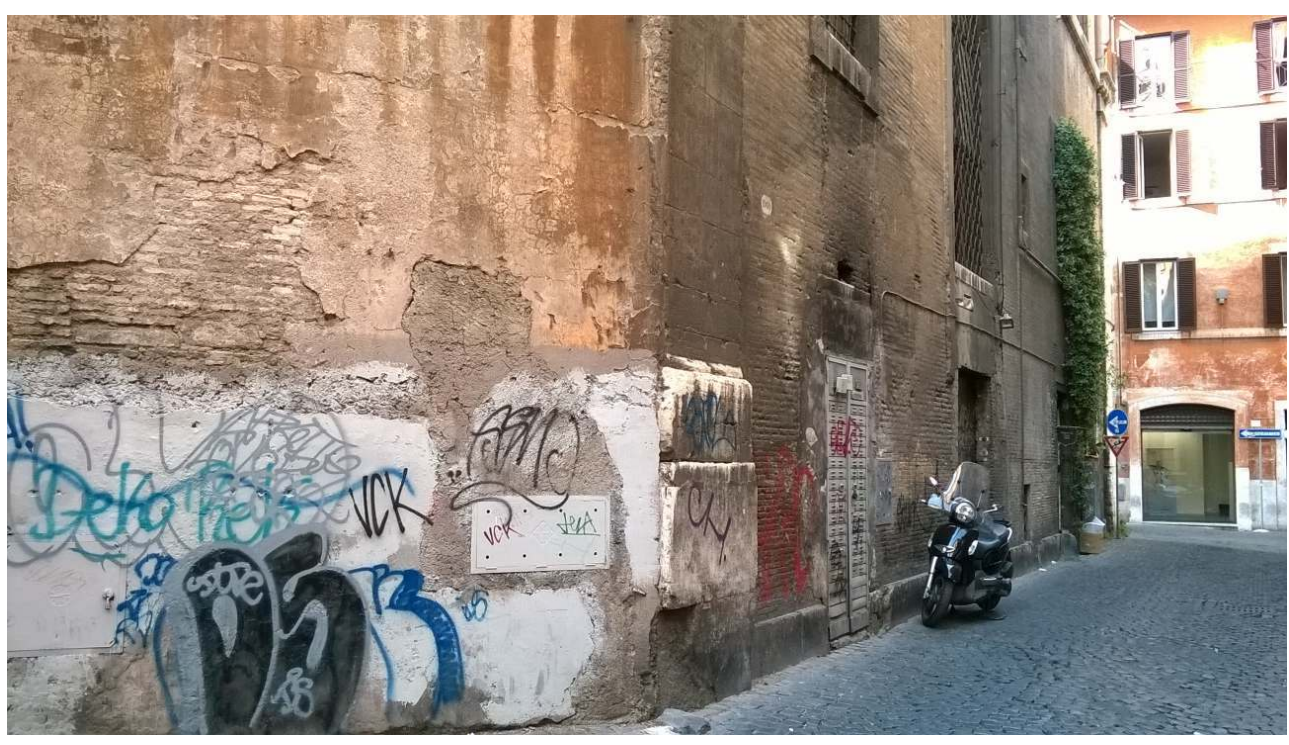

13 Il est né, car il est visible et tangible et il a un corps. En effet, toutes les choses de cette sorte sont sensibles et tout ce qui est sensible et appréhendé par l'opinion et la sensation est évidemment soumis au devenir et à la naissance ${ }^{4}$.

La rue est là, béante, et à la fois contrainte dans l'espace qu'elle propose à l'artiste. Elle contient le lieu, qui à son tour contient la place de l'œuvre. L'histoire joue un rôle prépondérant dans le choix de l'emplacement des figures. Ernest Pignon-Ernest n'en fait pas l'économie. Il fait jaillir le dessin des origines, le fait voir aux yeux de la rue, d'un carrefour, dans le recoin d'une façade ; le mur comme support matériel immédiat, partie intégrante d'un tout dramaturgique, d'une scénographie déjà existante. Un lieu de fiction, un élément d'illusion, qui finira par s'effacer, par disparaître en tant que tel. La trace laissée est imperceptible. Elle vit, transformée dans la mémoire, traversée par le souvenir, la pensée. Tous les éléments dramaturgiques de cette urbanité transcendée, les façades, les bruits, les sons, les mouvements des habitants, la présence des objets concourent à la théâtralité du lieu. L'espace public est libre d'accès. Il impose pourtant des contraintes techniques, logistiques à l'artiste, dans le maintien absolu de cette liberté de création. La rue surpeuplée, foisonnante de silhouettes humaines vivantes, peut vite devenir redondante et étouffer la figure collée jusqu'à l'écarter, l'exclure. Elle oblige l'artiste à agir de nuit, à la hâte, dans le plus parfait anonymat, pour se mettre à l'abri des regards parfois soupçonneux, inquisiteurs.

Dans la rue, les murs sont accolés, ils établissent des distances, des rapprochements. Ils contiennent et exhalent toutes les vies, les existences, leur commencement et leur fin. Ils ne disent pas la tragédie, le drame, ils sont le théâtre, la scène muette, ils offrent ces écarts entre le temps et l'espace à celui qui va le révéler. Les figures s'inscrivent dans cet 
espace entre le mur et la rue, dans cet infime pan à combler pour habiter ce lieu, sur une vibration rythmique entre le dehors et le dedans, entre l'ex-térieur et l'in-térieur, entre l'expression et l'impression. (Fig. 6)

Fig. 6

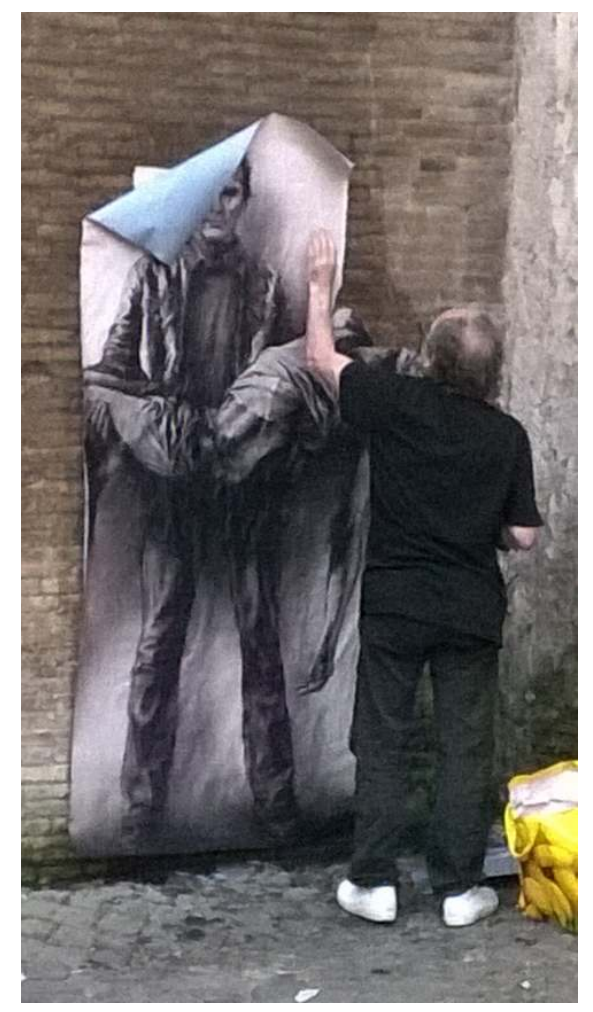

Les lieux sont des histoires fragmentaires et repliées, des passés volés à la lisibilité par autrui, des temps empilés qui peuvent se déplier, mais qui sont là, plutôt, comme des récits en attente et restent à l'état de rébus, enfin, des symbolisations enkystées, dans la douleur ou le plaisir du corps ${ }^{5}$.

Les corps dessinés viennent tracer à leur tour un lieu dans l'espace même. Corps de chair et d'os, d'encre et de papier. Tous se côtoient, se croisent, cohabitent, partagent le même lieu, la rue, dans un espace illimité, celui de la ville, qui l'enclot. D'aucuns sont vivants, mobiles, chantant et dansant, parlant ou criant; d'autres sont des personnages immobilisés dans un mouvement esquissé, figés dans leur geste, comme un arrêt sur image. Tous existent en réalité, dans notre imaginaire collectif. Ces corps viennent révéler, compléter, finir d'une certaine façon le monde des vivants. Silhouettes au fusain ou au crayon, elles se posent sur un mur, pour un temps plus ou moins compté, mais toujours suffisant pour s'inscrire dans le regard et la mémoire des passants qui capturent l'œuvre. CEuvre à peine née et déjà condamnée à disparaître. (Fig. 7) 
Fig. 7

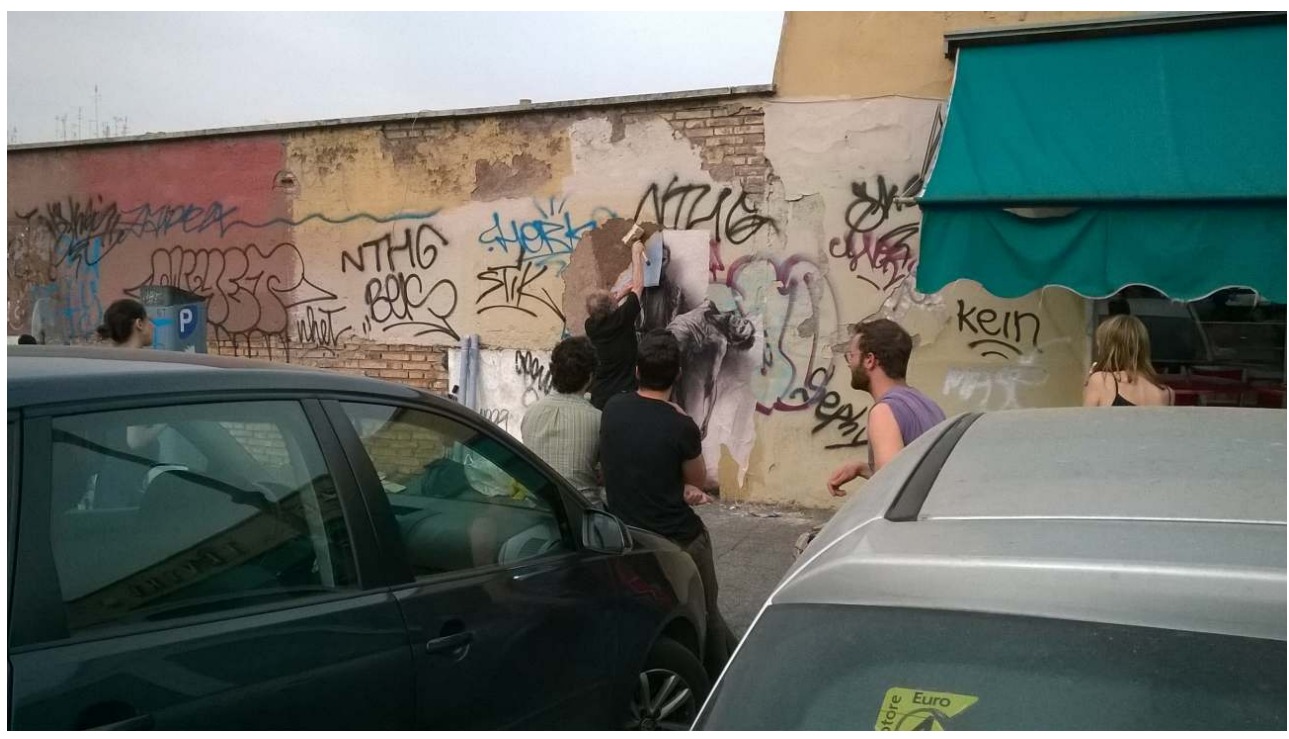

Et, en posant ses dessins, lèverait-il alors le voile de l'indicible qui masque les murs et tout ce qu'ils dissimulent? Il crée un espace supplémentaire, fictif, entre l'espace réel de la rue, mobile, changeant dans sa finitude géographique, dans son dessin dessein, et le plan du mur, matériel, minéral, en-dehors de sa symbolique originelle, livre de tous les écrits non écrits. Cet espace s'immisce dans un vaste ensemble, constitué de tous les éléments dramaturgiques instantanés, de l'univers sonore, des cris d'enfants, des voix de femmes qui s'interpellent d'une fenêtre à l'autre en étendant le linge, d'hommes qui s'apostrophent; des odeurs de la rue, de la ville qui montent des pavés, qui suintent des murs, des couleurs qui s'entrechoquent, des figures qui se déplacent en rythme et en vibration.

L'espace visuel et sonore est un espace sans cesse et infimement modifié, ancré dans une réalité quotidienne, urbaine. Il appartient aux lieux dans un temps défini. Cet espace se crée et se recrée de soi. L'espace fictif tel que le conçoit Ernest Pignon-Ernest en tant qu'origine d'une œuvre d'art, lieu de commencement, est un élément d'illusion rattaché au contexte signifiant, immédiatement identifiable par les référents culturels et historiques propres à chacun.

«Une seule illusion libre de tout lien : la possibilité d'exprimer indéfiniment la réalité à travers une succession de re-découvertes dramatiques ${ }^{6}$. "

21 L'orchestration de cette multitude de dispositifs scéniques, dramatiques, sans cesse renouvelés échappent à l'artiste. Les scénographies successives, dans lesquelles se créent et se recréent les œuvres à chaque fois réinventées, interpellées différemment, sont engendrées par un ballet continu de figures vivantes dans l'espace.

Les dessins insufflent aux murs cette part vitale, qui les extrait des ténèbres. Seule à pouvoir déplier l'univers et construire une référence sur un sens collectif, la figure joue un rôle symbolique, opère un renvoi immédiat à l'image mythique comprise dans le lieu, au travers du mur. 


\section{Rencontre du troisième type : la figure et le passant}

Théâtre humain, la rue est la scène, la platea, la place d'une rencontre fortuite, provoquée par les dessins d'Ernest Pignon-Ernest, entre les figures immobiles et les passants, libres de les voir ou de les ignorer sans le savoir.

Quel rapport entretient dès lors la figure avec les habitants du lieu ?

Le dessin-sujet est présenté au regard à dimension humaine, dans une scénographie élargie, dans un espace pourtant bordé par le support lui-même, le mur. L'artiste appréhende un morceau de réel dans toute sa complexité historique, géographique, sociale. Dans ces bouts de corps pris dans leur unité, il récupère une réalité forte de sa transfiguration. Il réhabilite le symbolique dans une présence mythique de la figure. L'image joue un rôle de révélateur, de perturbateur. Elle vient troubler le regard, accroche l'œil au passage, provoque un saisissement qui tient aussi de la fragilité, du caractère éphémère du support-papier. Le seul fait que l'image, livrée aux aléas du climat, pluies et vents, d'une main d'enfant tenté d'arracher le papier déjà légèrement décollé, puisse disparaître dans un temps très court, donne à l'inscription du sujet une urgence particulière. (Fig. 8)

Fig. 8

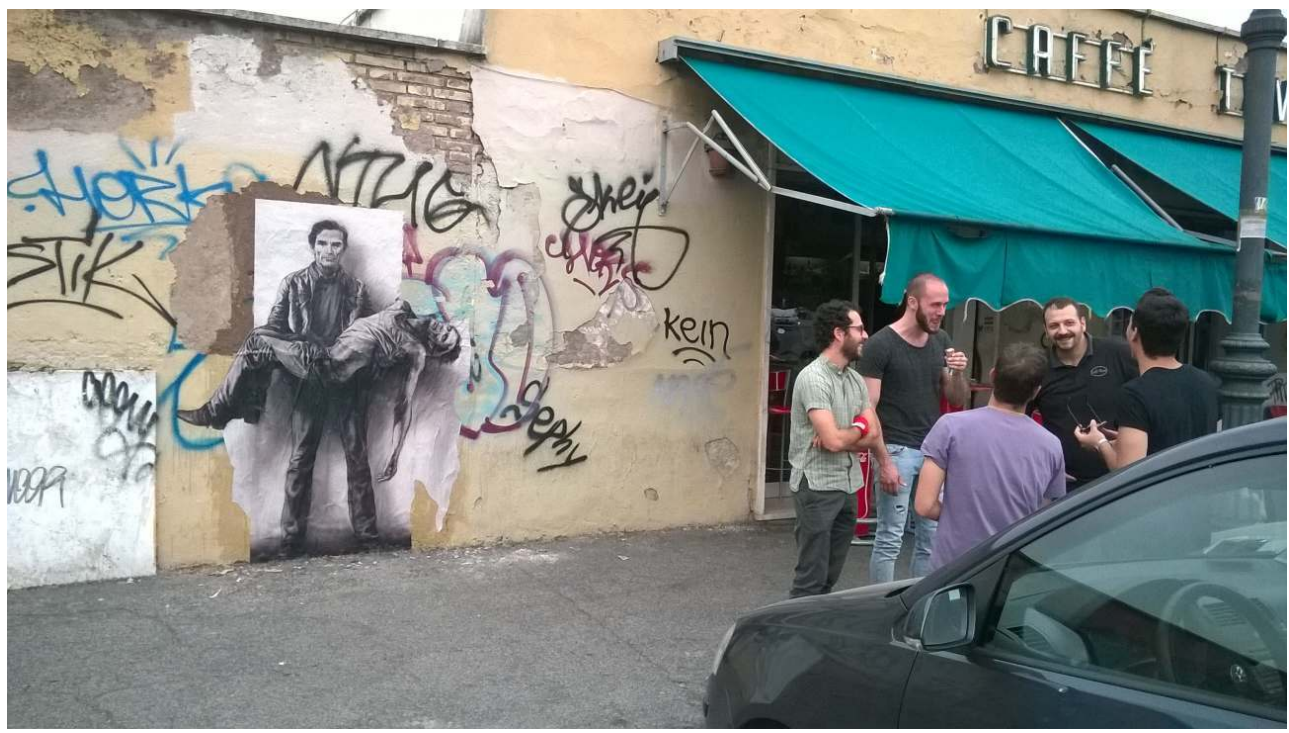

Au spectateur d'accepter cette expérience du vide, de se laisser aspirer et de traverser la forme, d'accéder à un ailleurs de la figure. Situation propice à une visite de l'imaginaire, sur la trace du fantasme, au souvenir rattrapé au détour d'une présence fugace de son absence encore plus aiguë. La figure s'absente irrémédiablement, disparaît. Plus tard, plus loin, une rencontre avec un autre dessin ravivera la mémoire des figures déjà croisées, disparues, puis ressuscitées.

Absence

déchirante présence.

Vague mémoire de toi

vagues souvenirs

qui troublent l'heure paisible

et le doux soleil. 
La poitrine douloureuse,

elle te porte

comme une pierre

légère ${ }^{7}$.

Un dessin collé d'Ernest Pignon-Ernest réveille la sensation immédiate que quelque chose d'important est en train d'avoir lieu, dans l'espace-temps signifié. Le signe a effracté le lieu, entrouvert une page-papier de son histoire, somme de toutes les histoires contenues. Le mur demeure, transfiguré, passage obligé pour accéder à un réel si souvent convoité. (Fig. 9)

Fig. 9

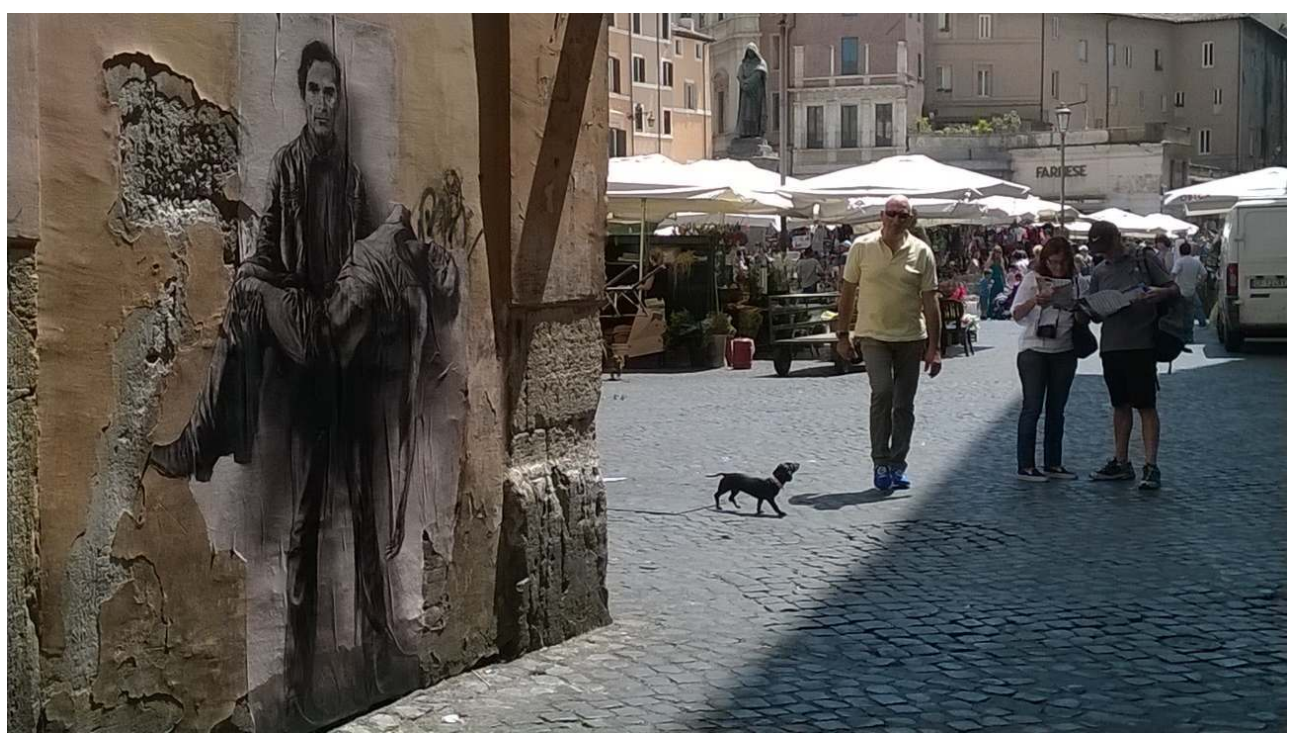

Sur les pierres, les dalles, les parois, les figures d'Ernest Pignon-Ernest tissent un voile, qui accentue le mystère à lui intrinsèque. La figure maintient cette part de non-dit dans l'ombre, dans un repli de son corps. Toute la ville suinte de ces figures, coffres-forts de tous les secrets qui affleurent aux yeux de ceux qui s'arrêtent et les scrutent. La figure, à son tour, tait au regard ce qu'elle enclot. Quand elle disparaît, subsiste l'empreinte, le lieu transformé, doublement signifiant.

La présence humaine de passage dans le lieu est tout aussi éphémère que la figure. L'apparition-présence est vouée à la disparition-absence, par le fait même du déplacement du sujet-regardant, par la détérioration orchestrée par les éléments naturels ou par une main volontairement destructrice...

Le lieu est cet espace circonscrit, qui permet de percevoir les en-jeux, ce qui se tisse, se trame, se lie, se délie entre les figures-rôles titres et les sujets-objets qui évoluent autour d'elles. Cette portion d'espace est transformée, ponctuellement détournée de son état précédent, par l'intrusion, l'installation d'une figure prévue et attendue, inouïe, qui n'a jamais été là. Le lieu transfiguré, traversé par cette figure inédite, est désormais habité autrement. Il convoque le regard, le dérange, l'extrait de ses habitudes. Il est cette différence dans la répétition. Le lieu ouvre sur un autre espace, l'espace dans l'espace, ramené du passé, augmenté, métonymié, distancié par la présence du dessin. L'espace est béant sur les images, qui métamorphosent, détourent, transforment un lieu commun, habituel, prêt à accueillir d'autres images. 


\section{Conclusion}

31 «Les lieux eux-mêmes sont porteurs d'un potentiel poétique, dramatique, suggestif. J'ai fait l'image ${ }^{8}$.»

32 Quand Ernest Pignon-Ernest pense un lieu, la ville, c'est toujours à partir d'une unité perdue, d'une globalité mise entre parenthèses. Il parcourt les rues, les avenues, les quais, en quête de la singularité de chaque espace, de bouts de lieu, de sa métaphore, pour lui restituer son entier, à partir de ce rien indicible, qu'est son origine. L'origine du lieu ne tient-elle pas dans la dramaturgie proposée au regard, à cette situation dont Ernest Pignon-Ernest fait œuvre en subjectivant le lieu par les figures ? La charge dramatique est inhérente au lieu, à son histoire, à ses histoires. Théâtre urbain à ciel ouvert, la rue accueille et rend dans son ensemble les éléments dramaturgiques visuels et sonores qu'elle contient. Les récits s'y superposent, s'entrechoquent, les paroles résonnent ou s'étouffent dans l'interstice des pierres. Des mots tus à jamais, des voix, des cris, des pleurs s'enfoncent chaque jour un peu plus, dans l'attente d'une figure qui les délivrera, les fera émerger au grand jour. Théâtre humain, scène du quotidien, mobile dans sa propre immobilité, mouvante, émouvante, trace anonyme de tant d'hommes, de femmes, d'enfants, de vieux, décor naturel de corps inanimés, tirés du silence, dans un face à face, avec les vivants. La figure habite désormais une scénographie géante, elle convoque, rapte le regard et met enfin à distance la présence.

S'interroger sur la pratique d'un artiste comme celle d'Ernest Pignon-Ernest, signifie partir sur ses traces dans les lieux qu'il habite poétiquement, en retrouver la force matricielle, entrevoir la richesse dramaturgique de chacun d'eux. Mais c'est aussi ouvrir sa pensée à leur caractère singulier, politique, social, mythologique, historique. Un lieunourrice pour filer la métaphore platonicienne. Un lieu-mémoire ravivé par des formes d'encre et de papier, ouvert aux regardeurs. (Fig. 10) 
Fig. 10

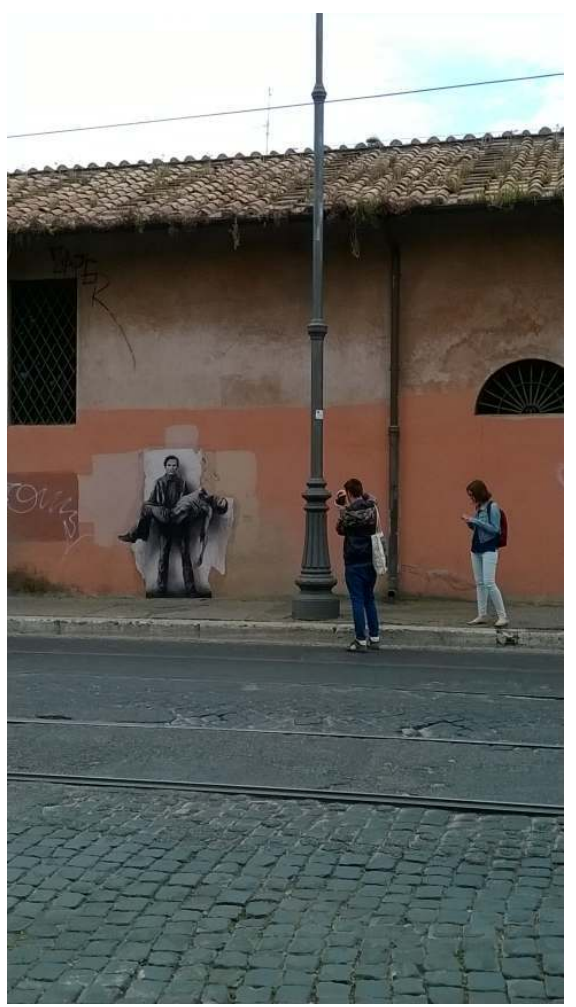

\section{BIBLIOGRAPHY}

\section{$\underline{\text { Livres }}$}

Bailly, J-C (2001) La ville à l'œuvre. Paris : Les éditions de l'Imprimeur.

Deleuze, G. (1968) Différence et répétition. Paris : Presses Universitaires de France.

Pasolini, P.P. (1976) L'expérience hérétique. Paris : Éditions Payot.

Pasolini, P.P. (1994) Qui je suis. Paris : Éditions Arléa.

Pasolini, P.P. (1997) Écrits sur la peinture. Paris : Éditions Carré.

Pignon-Ernest, E. (2008) Faire œuvre des situations. Avignon : Collection Entre- Vues, éditions Université d'Avignon.

Platon (1992) Le Timée. Paris : Éditions Gallimard.

Salignon, B. (2008) Où-L'art, l'instant, le lieu. Paris : Les éditions du Cerf.

Velter, A. (2014) Ernest Pignon-Ernest. Paris : Éditions Gallimard.

$\underline{\text { Film }}$

Pasolini, P.P. (1971) Le Décaméron. DVD France : Carlotta Films. 


\section{APPENDIXES}

Parcours romain : Pasolini, dans la succession d'une figure identique inlassablement déclinée.

De Pier Paolo Pasolini, Ernest Pignon-Ernest ne s'est jamais vraiment détaché. À Certaldo, en 1980, sur les remparts de la ville toscane, il colle sa figure en crucifié à l'envers, la tête prise dans un bandeau, allusion au personnage d'un disciple de Giotto que Pasolini incarne dans son film Le Décaméron. (Fig. 11)

Fig. 11

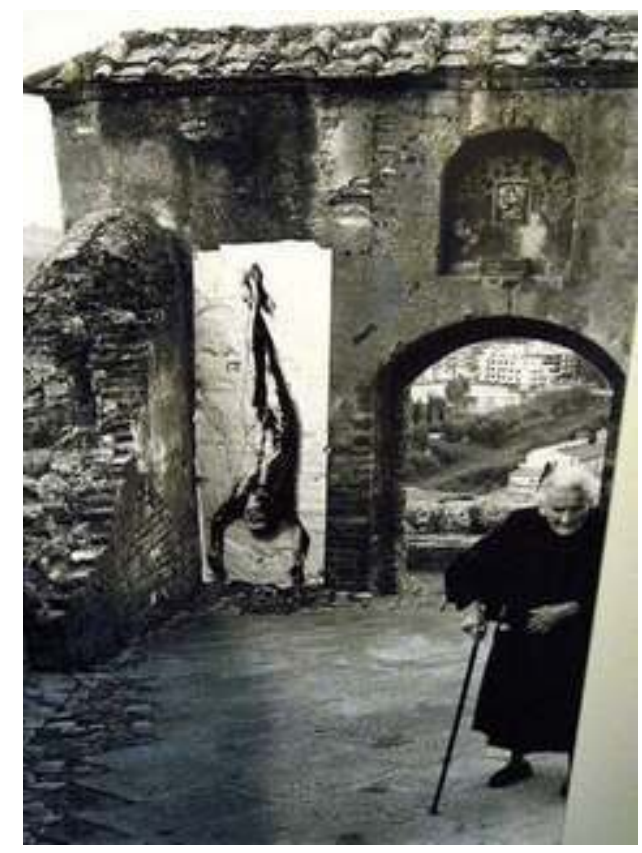

À Naples, en 1988, il associe Pasolini au Caravage, compagnons d'infortune à trois siècles d'écart, dans une citation empruntée à ce dernier. Il défie les interdits en collant la figure d'un chérubin tenant dans chaque main la tête tranchée de Pasolini et celle du Caravage juste au-dessus de la plaque Vietata l'affissione ${ }^{10}$.

Vingt-cinq ans plus tard, le soleil lèche la façade d'en face, un palais du XIXème siècle, qui s'étire vers un ciel voilé d'avril. D'une fenêtre tout en haut, une voix pointue déverse dans ce ruban d'asphalte une cascade de mots dans un napolitain serré. Une vespa pétarade, laisse un nuage de benzine derrière elle, coupant court à la conversation.

Dans le regard de la voisine d'en face resteront longtemps imprimées cette fenêtre ouverte, l'apparition d'un jeune homme, les deux trophées à la main. Elle le raconte aujourd'hui, encore sous le coup de l'émotion.

En mai 2015, Ernest Pignon-Ernest me convie à l'accompagner à Rome pour son projet Pasolini. J'ai participé jour et nuit à une vingtaine de collages dans les rues de la capitale italienne. J'en livre ci-dessous un récit complice.

Le jour n'est pas encore levé. Des poubelles éventrées bordent les rues sans trottoirs. Des gobelets poussés par le vent glissent sur les pavés humides. Rome ne s'éveille pas encore. 
Un homme se déplace furtivement, d'un pas mesuré, un cabas à la main, des rouleaux de papier sous le bras. Cet homme, c'est Ernest Pignon-Ernest. Il s'arrête au coin d'une rue, angle majeur du quartier de Trastevere. Des lieux encore hantés par Pier Paolo Pasolini la nuit finissante. Il hume l'air presque marin, tend l'oreille aux cris rauques des mouettes qui précèdent les éboueurs déjà à l'œuvre. Il inspire profondément avant de poser sac et rouleaux au pied du mur noirci de tags incompréhensibles, qui couvrent désormais toute la ville. Il passe la main sur la pierre, sur le crépi lépreux. Il constate les irrégularités, les anfractuosités. Il devra les combler avec les chutes de papier avant d'étirer la figure. Cette figure qu'il a longuement cherchée, inlassablement éprouvée, composée trait par trait dans son atelier : une pietà moderne, la figure de Pier Paolo Pasolini vivant avec dans les bras, son propre cadavre. Un cadavre vu et revu dans les dossiers judiciaires, sur les photographies prises à l'aube par la police, par les journalistes, diffusées en première page des journaux. Une figure immédiatement identifiable dans la ville papale, dans les cercles intellectuels et artistiques proches du poète.

Du sac, Ernest Pignon-Ernest sort un seau de colle, une brosse, des chiffons. Il enduit d'abord le mur, déroule ensuite le papier du bas vers le haut. Il étire la figure, bien droite et figée dans sa posture tragique. (Fig. 12) Ce sont désormais les passants qui l'animeront, qui la mettront en mouvement dans leur regard. Ils l'abandonneront à sa verticalité, à sa muralité, ou bien décideront de l'emmener avec eux, en photographie, dans leur I-Phone 4,5 ou 6 , dans un coin de leur mémoire à eux seuls accessible. (Fig. 13)

Fig. 12

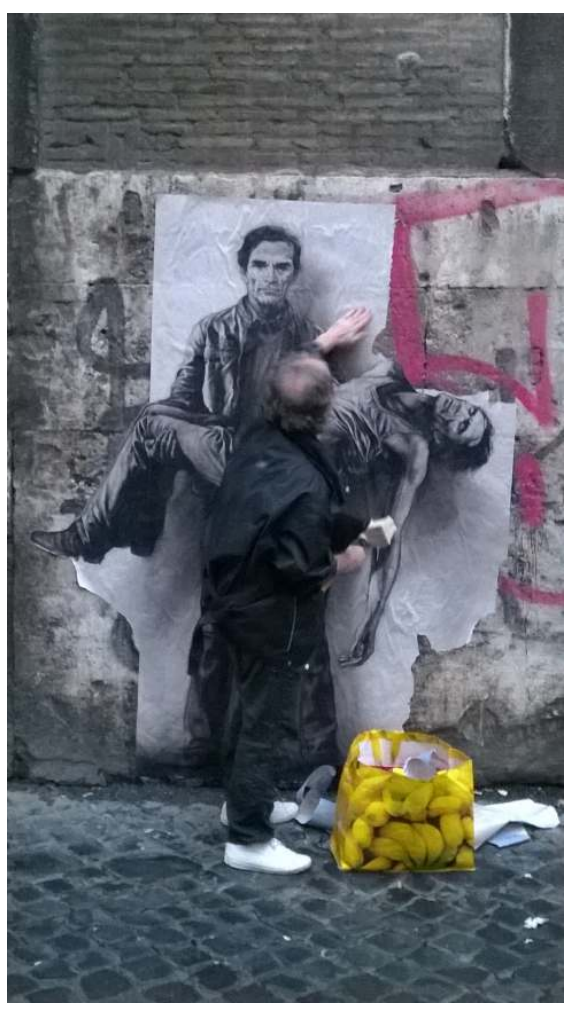


Fig. 13

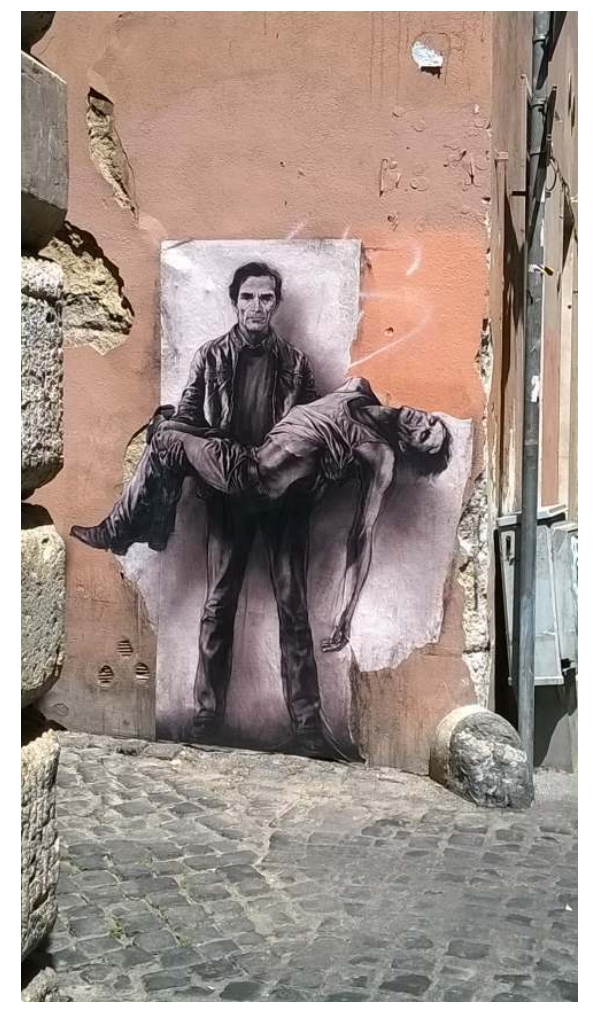

Ils ignorent souvent qui a fait œuvre. Si Ernest Pignon-Ernest ne signe pas ses collages, les figures se reconnaissent aisément et s'attribuent presque naturellement à son auteur. La curiosité du passant subjugué reste entière, accrue parfois par l'absence même de nom. Certains s'interrogent face à une telle puissance visuelle dans un espace qu'ils fréquentent quotidiennement. D'autres se laissent tout simplement surprendre, troubler, émouvoir. Le mystère est le fruit de l'apparition soudaine, inexpliquée, mystère augmenté par la disparition annoncée de cette œuvre éphémère. (Fig. 14)

Fig. 14

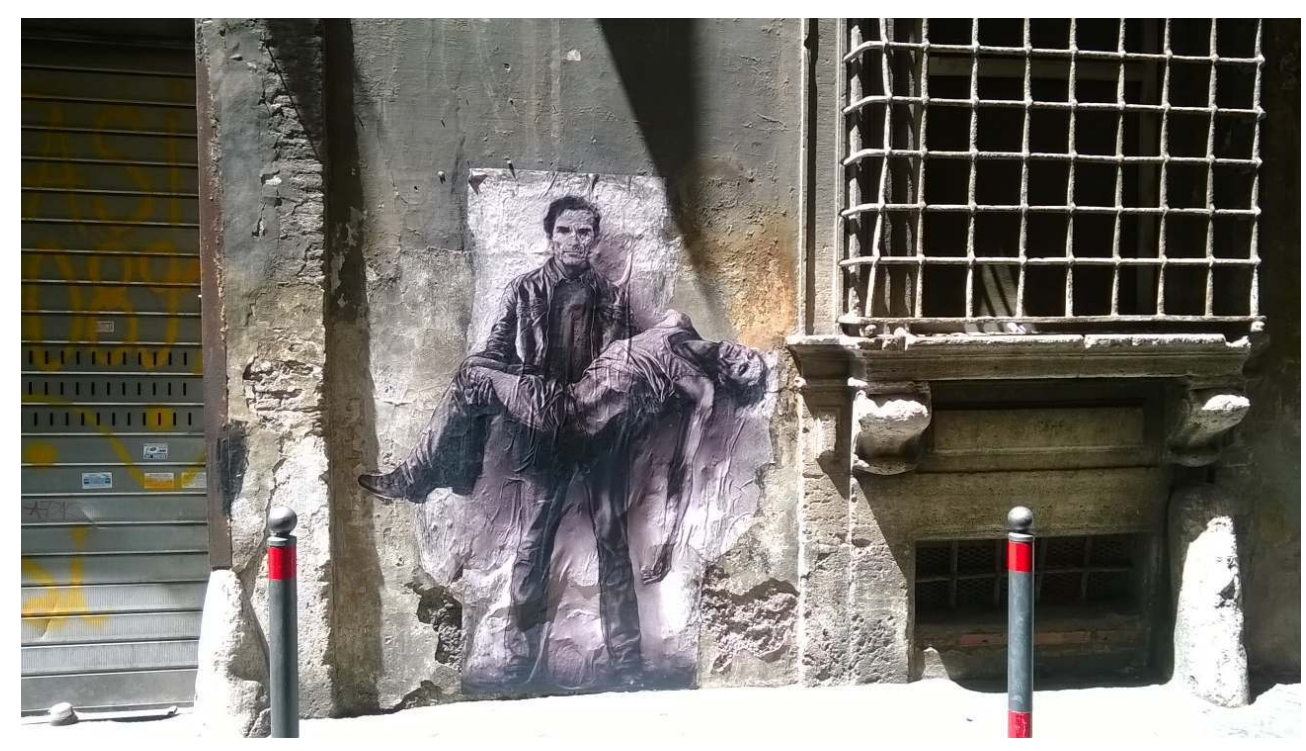


À Rome, en mai 2015, Ernest Pignon-Ernest a collé la figure de Pasolini en Pietà à différents endroits de la ville. Il est parti sur les traces du poète cinéaste, à la recherche des lieux d'écriture ou de tournage. Certains existaient encore, d'autres avaient disparu, engloutis sous des barres d'immeubles entourés de jardins publics, décorés de fresques commandées à des artistes pour enjoliver des lieux hostiles, comme à Rebibbia, autour de la prison, ou encore à Testaccio, au-delà du pont, le long du Tibre.

Lieu après lieu, dans cet espace, elles font la différence. Dans la mens momentanea ${ }^{11}$, l'esprit du moment, elles obéissent à la règle première de l'instantanéité. La distance s'opère d'un lieu à l'autre, jamais tout à fait dans l'oubli. La figure suivante apparaît dans les persistances de la précédente. La différence spatiale déplace et déguise, renvoie à l'autre, fait revenir. Le Même, le Seul, est atteint en traversant l'excès de l'Autre, dans le miroir de l'œil. Le processus de répétition individuelle, d'une figure identique à une autre figure identique, ailleurs, dans une dramaturgie unique de l'instant, variable dans le temps, glisse dans un processus de répétition universelle, dont la seule issue est la disparition, la Mort. La répétition répond à ce mouvement de rotation, de déplacement dans le temps synthétique du présent, en effaçant sur le moment l'avant et en taisant l'après. C'est l'apparition hic et nunc, dans un temps et un lieu uniques, une apparition théâtrale. Les parcours tracés par les figures sont horizontaux, longilignes et s'achèvent en une forme circulaire, avec un recommencement possible, dans la persistance des formes, dans la mémoire des figures.

La répétition, puissance du langage, sous-tend en permanence le trajet de la figure identique, réitère l'impression première, pourtant modifiée par la dramaturgie instantanée du lieu. En présence d'une figure identique à chaque fois renouvelée, le saisissement peut-il se reproduire?

Les figures se succèdent, se ressemblent, se répètent dans des lieux différents.

Chaque figure existe simultanément dans des regards différents. «La répétition ne change rien dans l'objet qui se répète, mais elle change quelque chose dans l'esprit de qui la contemple ${ }^{12}$. » Selon David Hume, il existe deux espèces de perceptions humaines : les impressions et les idées, pâles copies des impressions. L'impression, la première fois que la figure est perçue, est déconcertante. Elle frappe l'esprit qui la regarde, qui l'inscrit dans sa mémoire. La deuxième fois, la mémoire de la chose vue affaiblit, dédramatise le saisissement premier. A ceci près que chaque fois qu'Ernest Pignon-Ernest colle un dessin sur un mur, qu'il fait cohabiter une figure identique dans des lieux différents d'une même ville, il la fait apparaître dans une dramaturgie variable, qui ravive la première impression, provoque un temps d'arrêt, un saisissement peut-être amoindri, mais existant.

Dans la répétition déclinée des figures, les particolari (détails) seraient-ils le signifiant infime à même d'établir une nuance subtile dans le ressenti ? Une main abandonnée à même le sol, des doigts crispés qui relèvent le drapé d'une robe, qui ouvrent sur l'inattendue origine du monde, une bouche ébahie, des yeux écarquillés : des détails dans la globalité de la figure, qui déplacent le sens le temps du regard, puis le redépose et le laisse se fondre dans les contours. Dans l'imaginaire, l'être invisible, apparu tout à coup sous une forme visible, devient fantôme. Et se loge enfin dans un coin de la mémoire. (Fig. 15) 
Fig. 15

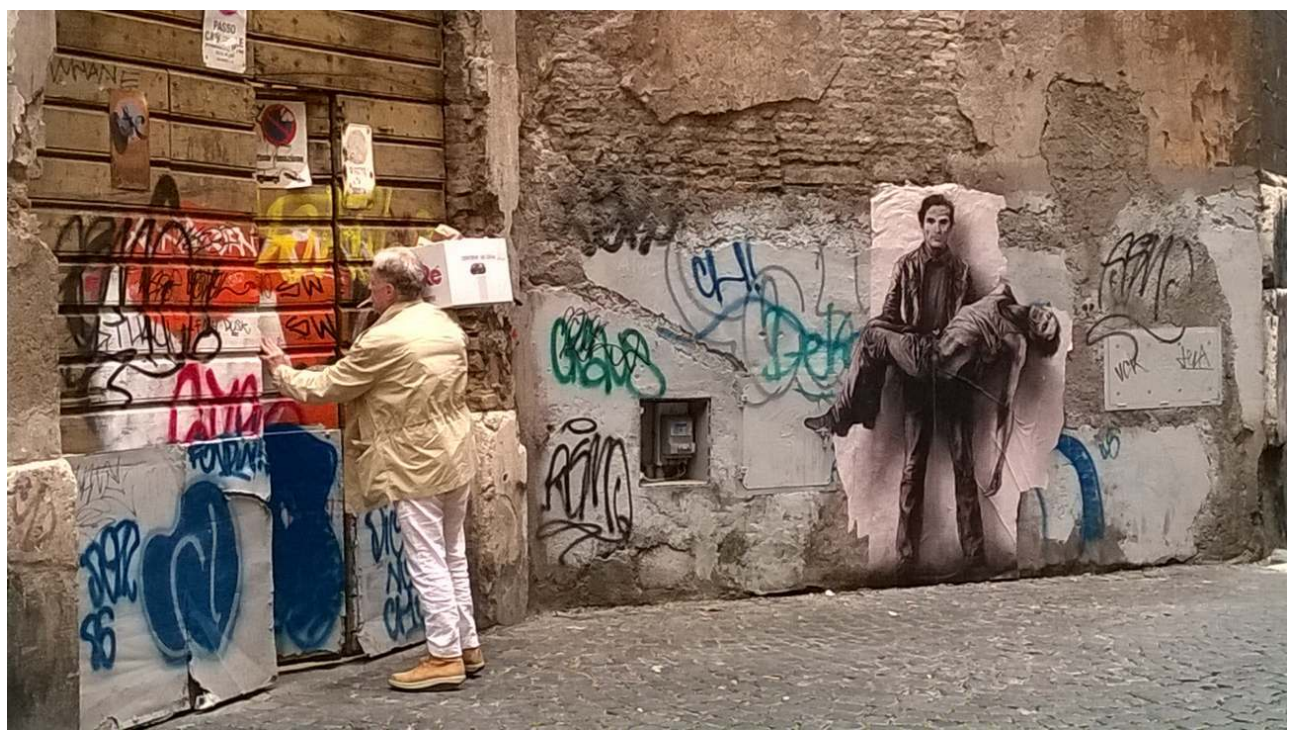

La déclinaison des figures ne vaut que par leur ensemble dans le parcours au cœur de la cité, en suivant les artères au rythme des pulsations sonores, vocales, mécaniques.

Les lieux successifs invitent à feuilleter l'album des figures dans les rues, à s'arrêter sur un dessin, à ouvrir son regard. Puis, il faudra tourner le coin comme on tourne la page. Dès le départ, la règle du jeu est claire : la figure prend et relâche l'homme ou la femme de la rue. Certains poursuivent leur chemin sans frémir, d'autres continuent de marcher, bouleversés, sans savoir encore qu'il y aura d'autres rencontres et d'autres figures. (Fig. 16)

Fig. 16

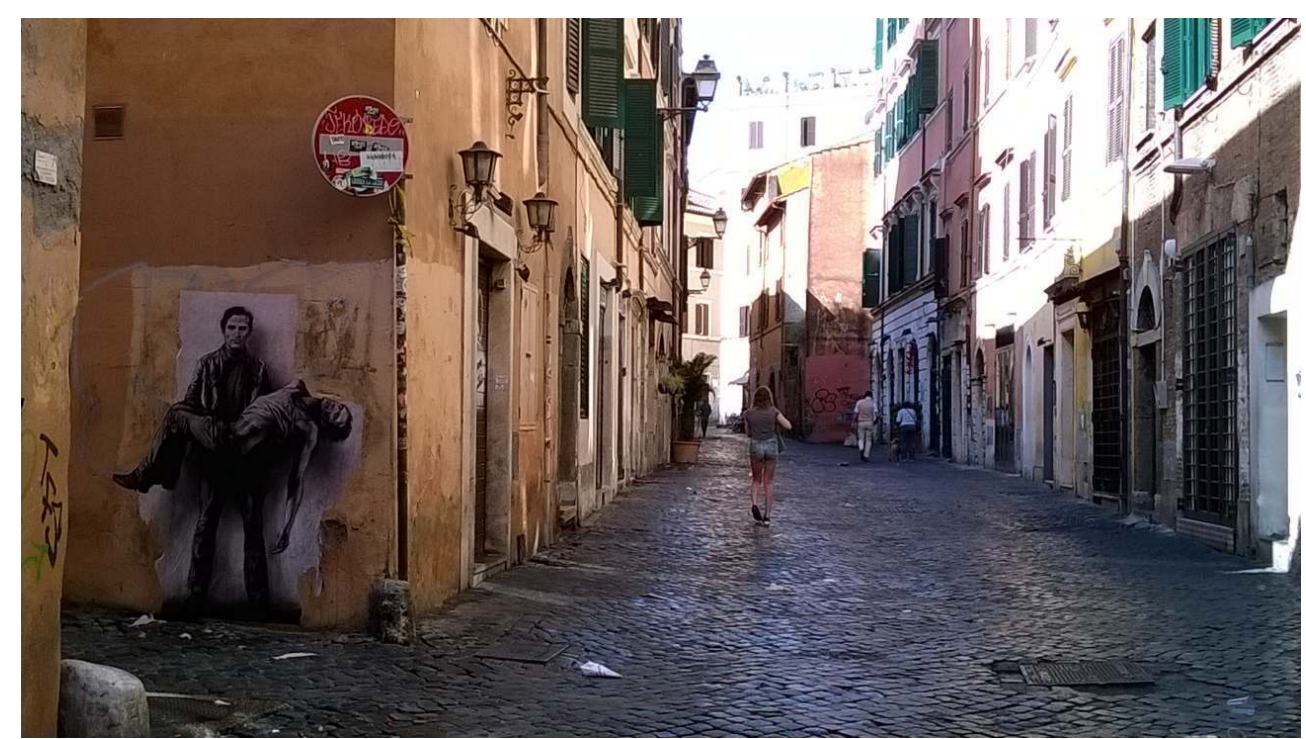




\section{NOTES}

1. PASOLINI, Pier Paolo : L'expérience hérétique, Paris, Éditions Payot, 1976, repris dans Qui je suis, Paris, Éditions Arléa, 1994, p.58.

2. SALIGNON, Bernard : Où - L'art, l'instant, le lieu, Paris, Les Éditions du Cerf, 2008, p.46.

3. BAILLY, Jean-Christophe : La ville à l'œuvre, Paris, Les éditions de l'Imprimeur, 2001, p.56.

4. PLATON : Le Timée, traduction Albert Rivaud, Paris, Gallimard, «Édition Les Belles Lettres », 1992, pp.183-184.

5. CERTEAU (de), Michel : L'invention du quotidien, phrase citée dans PIGNON-ERNEST, Ernest : Faire cuvre des situations, Avignon, éditions Université d'Avignon, Collection Entre-Vues, 2008, p.15.

6. PASOLINI, Pier Paolo : «Sur Roberto Longhi», in Écrits sur la peinture, Paris, Éditions Carré, «Arts\&Esthétique », 1997, présentation et traduction de Hervé Joubert-Laurencin.

7. BERTOLUCCI, Attilio: Sirio (1929), traduction en français par Karin Espinosa, entendu sur France Inter, le 16 septembre 2013 de $18 \mathrm{~h} 20$ à $19 \mathrm{~h}$.

Assenza,

più acuta presenza.

Vago pensier di te

vaghi ricordi

turbano l'ora calma

e il dolce sole.

Dolente il petto,

ti porta

come una pietra

leggera.

8. PIGNON-ERNEST, Ernest: Faire œuvre des situations, Avignon, éditions Université d'Avignon, Collection Entre-Vues, 2008, p.20.

9. PASOLINI, Pier Paolo : Le Décaméron, film adapté de l'œuvre éponyme de Boccace, France, 1971.

10. VELTER, André : Ernest Pignon-Ernest, Paris, Éditions Gallimard, 2014, p.181.

11. DELEUZE, Gilles : Différence et répétition, [1968], Paris, Presses Universitaires de France, «Coll. Épithémée », 2014, p.96.

12. HUME, David : «Traité de la Nature humaine » (1739), in Différence et répétition, ibid., p.96.

\section{ABSTRACTS}

Dans un théâtre urbain infini, depuis cinquante ans, la démarche d'Ernest Pignon-Ernest s'articule toujours en trois temps : le rapport de l'artiste au lieu, celui de la figure au lieu dans lequel il colle, et la relation qui s'instaure - ou pas - avec le passant-regardeur. Par la figuration, le lieu se révèle et se transfigure en un autre lieu, individuel et collectif. En annexe, une expérience personnelle et singulière apporte une dimension concrète à cette approche esthétique. 
In un teatro urbano infinito, da ormai cinquant'anni, il cammino di Ernest Pignon-Ernest si svolge sempre in tre tempi : il rapporto dell'artista con il luogo, il rapporto della figura con codesto luogo dove lui incolla, poi la relazione che nasce - o no - con il passante-guardante. Attraverso la figurazione, il luogo si svela trasfigurandosi in un altro luogo, individuale e collettivo. In allegato, un'esperienza personale e singolare dà a quest'approccio estetico una dimensione concreta.

INDEX

Geographical index: France, Italie

Mots-clés: esthétique, poétique, street art

Chronological index: XXème siècle, XXIème siècle

\section{AUTHOR}

\section{KARIN WACKERS-ESPINOSA}

Université Paul-Valéry, Montpellier III, RIRRA21, Esthétique, doctorante sous la direction de Marie Bourjea 